\title{
Proceso de envejecimiento, ejercicio y fisioterapia
}

\author{
Aging, exercising and physical therapy
}

\section{MSc. Nancy Stella Landinez Parra,' Dra. Katherine Contreras Valencia,' Dr. Ángel Castro Villamil"}

' Facultad Medicina, Universidad Nacional de Colombia. Bogotá, Colombia.

"Universidad del Rosario. Bogotá, Colombia.

\section{RESUMEN}

Objetivo: describir los beneficios de la actividad física y del ejercicio regular en la población mayor.

Métodos: se realizó una búsqueda en las bases de datos Science Direct, Springer J ournal, Springer books y Pubmed, se contó además con la ayuda del buscador de información científica Scirus. Se utilizaron los siguientes términos de búsqueda: envejecimiento, fisioterapia, actividad física, ejercicio físico, adulto mayor. Los artículos examinados incluyeron revisiones, artículos de investigación y capítulos de libros.

Síntesis de los datos: se ofrece la fundamentación de los principales procesos propios del envejecimiento, los cambios estructurales que este genera y sus consecuencias fisiológicas o fisiopatológicas. Se incluye el termino "Envejecimiento Activo", propuesto por la OMS, que conlleva estrategias de promoción de salud y prevención de las deficiencias y las discapacidades e involucra programas de ejercicio físico o actividad física practicados de manera regular. La fisioterapia ayuda a promover, prevenir, curar y recuperar la salud de los individuos, varios de sus programas están destinados a tratar los problemas de discapacidad generados por enfermedades como el parkinson, alzheimer, artritis, fracturas, osteoporosis, caídas y problemas cardiorrespiratorios. Promueven el movimiento y la independencia mediante ejercicios, técnicas de relajación, manejo del dolor, cuidados cardiorrespiratorio y otros.

Conclusiones: someter el organismo a un programa de entrenamiento planificado, contribuye a mejorar la capacidad funcional de múltiples sistemas orgánicos; los programas de ejercicio dirigidos a la población mayor pueden generar un ahorro en los costos sanitarios por disminución de caídas, accidentes cerebrovasculares, infartos del miocardio o enfermedades crónicas propias del proceso de envejecimiento. 
Palabras clave: envejecimiento, fisioterapia, actividad física, ejercicio físico, adulto mayor.

ABSTRACT

Objective: to describe the benefits of physical activity and regular exercising for the older population.

Methods: a search was made in Science Direct, Springer Journal, Springer books and Pubmed databases, with the support of scientific information searcher Scirus. The terms for the search were aging, physical therapy, physical activity, physical exercising and older people. The analyzed articles covered reviews, research papers, and book chapters.

Results: the fundamentals for the main processes inherent to aging, the structural changes that they generate and their physiological or physiopathological consequences were provided. The term "active aging", suggested by the WHO, was included. It implies several strategies for the health promotion and for the prevention of deficiencies and disabilities, and also involves regular physical exercising or activity programs. Physical therapy helps to promote health, to prevent and cure diseases and to recover the individual's health. Some of its programs are aimed at treating disability problems from diseases such as Parkinson, Alzheimer's, arthritis, bone fractures, osteoporosis, falls and cardiorespiratory disorders, and at encouraging movement and self-sufficiency through exercises, relaxation, pain management, cardiorespiratory care and others.

Conclusions: a planned training program for the body contributes to improve the functional capacity of multiple organ systems; additionally, the exercising programs aimed at the older population may bring a reduction of health costs by preventing falls, strokes, myocardial infarction or chronic diseases inherent to aging.

Key words: aging, physical therapy, physical activity, physical exercise, older people.

Envejecer es como escalar una gran montaña: mientras se sube las fuerzas disminuyen, pero la mirada es más libre, la vista más amplia y serena.

I NGMAR BERGMAN

\section{NTRODUCCI ÓN}

El envejecimiento humano es un fenómeno universal e inevitable. La esperanza media de vida ha mejorado en el último siglo. Actualmente, alrededor del $7 \%$ de la población mundial es de 65 años y más de edad. En los países desarrollados, este porcentaje es aún mayor ( $15 \%$ ) y continua creciendo. ${ }^{1}$ El envejecimiento en los seres humanos se asocia con una pérdida de la función neuromuscular y del rendimiento, en parte relacionadas con la reducción de la fuerza y la potencia muscular, ${ }^{2,3}$ causada por una pérdida de la masa de los músculos esqueléticos (sarcopenia) ${ }^{4}$ y los cambios en 
la arquitectura muscular. Esta disminución en la fuerza y la potencia muscular, junto con otros factores como el envejecimiento del sistema nervioso somatosensorial y motor, ${ }^{5}$ tiene implicaciones funcionales, tales como disminución en la velocidad al caminar, ${ }^{6}$ aumento del riesgo de caídas, ${ }^{7}$ y una reducción de la capacidad para llevar a cabo las actividades de la vida diaria (AVD). Todo esto contribuye a una pérdida de la independencia y a una reducción en la calidad de vida de las personas. ${ }^{6}$

En los Estados Unidos, el $60 \%$ de las personas consideradas mayores no participan regularmente en actividades físicas, ${ }^{8-11}$ y en otros países desarrollados, entre el $30-80 \%$ de las personas son físicamente inactivas. ${ }^{12-14}$

La inactividad física es un importante factor de riesgo para muchas condiciones y problemas crónicos de salud, tales como las enfermedades cardiovasculares, la hipertensión, la obesidad, la osteoporosis, la diabetes mellitus y de salud mental. ${ }^{11,15-}$ 18

El presente trabajo pretende plantear la fundamentación de los principales procesos propios del envejecimiento, los cambios estructurales que este genera y sus consecuencias fisiológicas o fisiopatológicas. De igual forma se plantean de manera general los beneficios de un programa de actividad física o ejercicio dirigido en esta población.

\section{MÉTODOS}

Para recopilar la información se realizó una búsqueda de literatura en las bases de datos Science Direct, Springer J ournal, Springer books y Pubmed, se contó además con la ayuda del buscador de información científica Scirus. Se utilizaron los siguientes términos de búsqueda: envejecimiento, fisioterapia, actividad física, ejercicio físico, adulto mayor. Los artículos que se examinaron incluyeron revisiones del tema, artículos de investigación y capítulos de libros clasificados como fuentes primarias y secundarias. Una vez obtenida la información se procedió a realizar un análisis crítico de su contenido y se estableció su importancia y pertinencia con el proceso de envejecimiento, la fisioterapia y el ejercicio físico.

\section{SÍ NTESI S DE LOS DATOS}

\section{El envejecimiento}

Se ha considerado que el envejecimiento es un proceso multifactorial, (biológico, psicoespiritual, social), pero fundamentalmente biológico. La importancia genética en la regulación del envejecimiento biológico es demostrada por la longevidad característica para cada especie. Sin embargo, la herencia solamente influencia alrededor del $35 \%$ de la variabilidad del tiempo total de sobrevida de una especie, mientras que los factores medioambientales son responsables del $65 \%$ restante. ${ }^{19}$

El envejecimiento es un proceso fisiológico que comienza en la concepción y ocasiona cambios característicos de la especie durante todo el ciclo de la vida. Tales cambios producen una limitación a la adaptabilidad del organismo en relación con el medio. Sin embargo, es importante distinguir el envejecimiento como un proceso: el proceso de envejecimiento. La OMS en su documento "Hombres, envejecimiento y salud" considera esta diferencia. El envejecimiento como proceso (envejecimiento normal)

http://scielo.sld.cu 
representa los cambios biológicos universales que se producen con la edad y que no están afectados por la influencia de enfermedades o del entorno. De esta forma, no todos los cambios relacionados con la edad tienen consecuencias clínicas negativas. Por el contrario, el proceso de envejecimiento está muy influenciado por los efectos de los estados del entorno, del estilo de vida y de las enfermedades, que a su vez, están relacionados con el envejecimiento o cambian por su causa pero que no se deben al envejecimiento en sí. ${ }^{20}$

El ritmo en esos cambios se produce en los distintos órganos de un mismo individuo o en distintos individuos en forma desigual. El punto de corte para definir el envejecimiento, a los efectos estadísticos, es la edad de 60 años, pero para los efectos biológicos, este punto lo marca la declinación de las actividades somáticas y mentales. A este respecto la OMS utiliza categorías que empiezan a la edad de 65 años. ${ }^{20}$

Características del envejecimiento. El envejecimiento presenta características inherentes y bien definidas en todos los seres vivos, entre ellas:

- Universal: esto es, que es propio de todos los seres vivos.

- Progresivo: al trascurrir la vida se producen efectos sobre el organismo, que al acumularse originan los cambios propios del envejecimiento.

- Irreversible: a diferencia de las enfermedades, no puede detenerse ni revertirse.

- Heterogéneo e individual: cada especie tiene una velocidad característica de envejecimiento, pero la velocidad de declinación funcional varía enormemente de sujeto a sujeto, y de órgano a órgano dentro de la misma persona.

- Deletéreo: lleva a una progresiva pérdida de función. Se diferencia del proceso de crecimiento y desarrollo en que la finalidad de este último es alcanzar una madurez en la función.

- Intrínseco: no es debido a factores ambientales modificables. En los últimos 20 años se ha observado un aumento progresivo en la expectativa de vida de la población, la máxima sobrevida del ser humano se manifiesta alrededor de los 118 años. A medida que se ha logrado prevenir y tratar mejor las enfermedades y se han mejorado los factores ambientales, la curva de sobrevida se ha hecho más rectangular. Se observa que una mayoría de la población logra vivir hasta edades muy avanzadas con buena salud y muere generalmente alrededor de los 80 años.

Todo lo anterior nos permite entender que actualmente se puede contar con una mayor tasa de sobrevida, pero que definitivamente esta se asocia a diferentes cambios físicos y fisiológicos.

Cambios físicos del envejecimiento. El envejecimiento produce alteraciones sistémicas cuando la mayoría de los órganos y tejidos van disminuyendo su actividad. Estas modificaciones comprenden la reducción de la flexibilidad de los tejidos, la pérdida de células nerviosas, el endurecimiento de los vasos sanguíneos y la disminución general del tono corporal. Diversas causas se han asociado a este deterioro, entre ellas, las de índole genético, los cambios en la actividad metabólica celular o en los procesos bioquímicos, las alteraciones hormonales y las condiciones ambientales. Varias de ellas son alteraciones que ocurren en los distintos aparatos y sistemas como resultado del proceso de envejecimiento. ${ }^{21}$ 
El recuadro recopila los cambios estructurales en los diferentes aparatos y sistemas, cada uno con sus consecuencias fisiológicas o fisiopatológicas, propias del proceso mismo de envejecimiento.

Recuadro. Principales cambios estructurales y sus consecuencias fisiológicas o fisiopatológicas

en el proceso de envejecimiento

\begin{tabular}{|c|c|}
\hline Cambios estructurales & $\begin{array}{l}\text { Consecuencias fisiológicas } \\
\text { o fisiopatológicas }\end{array}$ \\
\hline $\begin{array}{l}\text { Composición corporal: } \\
\text { - Disminución de agua corporal. } \\
\text { - Disminución del tamaño de los órganos. } \\
\text { - Aumento relativo de la grasa corporal. }\end{array}$ & $\begin{array}{l}\text { Resistencia disminuida a la deshidratación. } \\
\text { Alt Alteración en la distribución de } \\
\text { fármacos. }\end{array}$ \\
\hline $\begin{array}{l}\text { Sistema tegumentario: } \\
\text { - Disminución del recambio de células } \\
\text { - epidérmicas. } \\
\text { - Atrofia dermoepidérmica y subcutánea. } \\
\text { - Disminución del número de melanocitos. } \\
\text { - Atrofia de folículos pilosos y glándulas } \\
\text { sudoríparas. } \\
\text { - Disminución de actividad de glándulas } \\
\text { - } \text { sebáceas. } \\
\text { Disminución de vasculatura dérmica y asas } \\
\text { capilares. }\end{array}$ & $\begin{array}{l}\text { Arrugas cutáneas y laxitud. } \\
\text { Fragilidad capilar. } \\
\text { Telangiectasias. } \\
\text { Susceptibilidad a úlceras de decúbito. } \\
\text { Xerosis cutánea. } \\
\text { Queratosis actínica. } \\
\text { Encanecimiento y alopecia. }\end{array}$ \\
\hline $\begin{array}{l}\text { Aparato cardiovascular: } \\
\text { Disminución del número de células } \\
\text { miocárdicas y de la contractilidad. } \\
\text { - Aumento de resistencia al llenado } \\
\text { ventricular. } \\
\text { - Descenso de actividad del marcapasos AV, } \\
\text { velocidad de conducción y sensibilidad de los } \\
\text { barorreceptores. } \\
\text { - Rigidez de las arterias. } \\
\text { - Descenso del gasto cardíaco y del flujo } \\
\text { sanguíneo de la mayoría de los órganos. }\end{array}$ & $\begin{array}{l}\text { Disminución de la reserva cardíaca. } \\
\text { Escasa respuesta del pulso con el ejercicio. } \\
\text { Arritmias. } \\
\text { Aumento de la presión diferencial del pulso. } \\
\text { Aumento de la presión arterial. } \\
\text { Respuesta inadecuada al ortostatismo. } \\
\text { Síncopes posturales. }\end{array}$ \\
\hline $\begin{array}{l}\text { Aparato respiratorio: } \\
\text { Disminución de la distensibilidad de la pared } \\
\text { torácica y pulmonar. } \\
\text { - Pérdida de septos alveolares. } \\
\text { - Colapso de las vías aéreas y aumento del } \\
\text { volumen de cierre. } \\
\text { Disminución de la fuerza de la tos y } \\
\text { aclaramiento mucociliar. }\end{array}$ & $\begin{array}{l}\text { Disminución de la capacidad vital. } \\
\text { Aumento del volumen residual y de la } \\
\text { diferencia alvéolo arterial de oxígeno. } \\
\text { Aumento del riesgo de infecciones } \\
\text { y broncoaspiración. }\end{array}$ \\
\hline $\begin{array}{l}\text { Aparato renal: } \\
\text { - Descenso absoluto del número de nefronas, } \\
\text { disminución del peso renal. } \\
\text { - Descenso del tono vesical y del esfínter. } \\
\text { - Disminución de la capacidad de la vejiga. }\end{array}$ & $\begin{array}{l}\text { Reducción del filtrado glomerular (la cifra de } \\
\text { creatinina se mantiene por descenso de } \\
\text { producción). } \\
\text { Disminución en la habilidad de } \\
\text { concentración } \\
\text { y máxima capacidad de reabsorción de la }\end{array}$ \\
\hline
\end{tabular}




\begin{tabular}{|c|c|}
\hline $\begin{array}{l}\text { Hipertrofia prostática en hombres y descenso } \\
\text { del tono de la musculatura pélvica en } \\
\text { mujeres. }\end{array}$ & $\begin{array}{l}\text { glucosa. } \\
\text { Incontinencia. }\end{array}$ \\
\hline $\begin{array}{l}\text { Aparato gastrointestinal } \\
\text { Boca: } \\
\text { - Disminución de la producción de saliva. } \\
\text { Erosión de dentina y del esmalte. } \\
\text { - Reabsorción de la raíz y migración apical de } \\
\text { estructuras de soporte del diente. } \\
\text { Esófago: } \\
\text { - Disminución del peristaltismo. } \\
\text { Estómago e intestino: } \\
\text { - Disminución de la secreción de ácido y } \\
\text { enzimas. } \\
\text { Colon y recto: } \\
\text { - Disminución del peristaltismo. }\end{array}$ & $\begin{array}{l}\text { Pérdida de piezas dentarias. } \\
\text { Tránsito esofágico prolongado. } \\
\text { Reflujo esofágico. } \\
\text { Disfagia. } \\
\text { Poliposis gástrica y metaplasia intestinal. } \\
\text { Constipación y diverticulosis. } \\
\text { Incontinencia fecal. }\end{array}$ \\
\hline $\begin{array}{l}\text { Sistema nervioso: } \\
\text { - Pérdida neuronal variable. } \\
\text { - Disminución de conexiones interdendríticas } \\
\text { - y de neurotransmisión colinérgica. } \\
\text { - Disminución del flujo sanguíneo cerebral. } \\
\text { - Alteración en los mecanismos de control de } \\
\text { temperatura y de la sed. }\end{array}$ & $\begin{array}{l}\text { Alteraciones intelectuales. } \\
\text { Lentitud y escasez de movimientos. } \\
\text { Hipotensión postural, mareos, caídas. } \\
\text { Reaparición de reflejos primitivos. } \\
\text { Hipo e hipertermia. } \\
\text { Deshidratación. }\end{array}$ \\
\hline $\begin{array}{l}\text { Sentidos } \\
\text { Vista: } \\
\text { - Fisiología alterada del vítreo y retina. } \\
\text { - Degeneración macular. Trastorno de } \\
\text { coloración, rigidez y tamaño del cristalino. } \\
\text { Oído: } \\
\text { - Disminución de la función de células } \\
\text { sensoriales en el aparato vestibular. } \\
\text { Gusto y olfato: } \\
\text { - Descenso en número y función de papilas } \\
\text { gustativas y células sensoriales olfatorias. }\end{array}$ & $\begin{array}{l}\text { Disminución de agudeza visual, campos } \\
\text { visuales y velocidad de adaptación a la } \\
\text { oscuridad. } \\
\text { Trastorno en la acomodación y reflejos } \\
\text { pupilares. } \\
\text { Alta frecuencia de cataratas, astigmatismo } \\
\text { y miopía. } \\
\text { Disminución de la audición (altas } \\
\text { frecuencias), discriminación de sonidos y } \\
\text { alteraciones del equilibrio. } \\
\text { Disminución de la satisfacción } \\
\text { gastronómica. }\end{array}$ \\
\hline
\end{tabular}


Tacto:

Disminución de agudeza táctil y de temperatura.

- Receptores de dolor intactos.

Aparato locomotor

Estatura:

Acortamiento de la columna vertebral por estrechamiento del disco.

- Cifosis.

Huesos:

Los huesos largos conservan su longitud. Pérdida universal de masa ósea.

Articulaciones:

- Disminución de la elasticidad articular.

Descenso progresivo de altura.

Osteoporosis.

Colapso vertebral y fractura de huesos largos con traumas mínimos.

Limitación articular.

Pérdida de fuerza muscular progresiva.

Disminución de la eficacia mecánica del músculo.

Degeneración fibrilar del cartílago articular, con atrofia y denudación de la superficie.

Músculos :

Disminución del número de células musculares.

Aumento del contenido de grasa muscular.

Adaptado de: Ob. cit. 19.

Cambios en la función cognitiva. La función cognitiva de un individuo es el resultado del funcionamiento global de sus diferentes áreas intelectuales, incluyendo el pensamiento, la memoria, la percepción, la comunicación, la orientación, el cálculo, la comprensión y la resolución de problemas. Esta función cambia con la edad. Actualmente, un trastorno caracterizado por un deterioro cognitivo adquirido de suficiente gravedad como para afectar al funcionamiento social y profesional se entiende como demencia. ${ }^{22}$ El impacto de la demencia es de gran alcance. No solo afecta la salud y el bienestar del paciente, sino que también está asociado a una pesada carga para el cuidador, un aumento del uso de los servicios sanitarios y necesidades de cuidado a largo plazo.

Si bien algunos individuos envejecen "exitosamente", es decir muchas de sus funciones cognitivas permanecen igual que en su juventud, la mayoría sufre la disminución de algunas esferas cognitivas tales como las de aprender nueva información y ejecutar funciones motoras rápidas, mientras que otros tienen, por ejemplo, la enfermedad de Alzheimer que deteriora severamente su funcionamiento cognitivo. ${ }^{23}$

Los pacientes con deterioro cognitivo requieren mayor supervisión por sus cuidadores, no colaboran en su rehabilitación y utilizan un mayor número de recursos 
sociosanitarios. Por ello, conocer el nivel cognitivo del paciente geriátrico es importante a la hora de planificar sus cuidados y tomar decisiones, ya que un apropiado manejo puede mejorar sustancialmente la calidad de vida y reducir el desarrollo de complicaciones. ${ }^{23}$

La disminución de las funciones cognitivas durante el envejecimiento es uno de los aspectos que suele vivirse como una gran amenaza para el bienestar e incluso para la integridad personal. Muchos adultos mayores, por ejemplo, refieren la pérdida de memoria con mayor angustia, que un dolor crónico, o toleran peor la falta de relación familiar que un determinado proceso de enfermedad. ${ }^{24}$ Teniendo en cuenta la estrecha relación entre estos aspectos y la lenta evolución con que suelen producirse las modificaciones en esta área, resulta difícil generalizar el alcance de las pérdidas ya que los adultos mayores irán adaptándose poco a poco e incluso ensayarán y pondrán en práctica estrategias alternativas para los déficits que van apareciendo. ${ }^{24}$

La pérdida de memoria reciente parece ser el signo general característico de los cambios cognitivos, durante el envejecimiento. A la persona le resulta difícil evocar sucesos recientes y sufre además pequeños olvidos. Diferentes factores se interrelacionan con esta pérdida de memoria, aunque no se conocen las causas exactas ni tampoco el alcance de esta interacción, que abarcan desde los cambios neurológicos y circulatorios que afectan la función cerebral, la oxigenación y la nutrición celular, hasta la motivación, la pérdida de interés por el entorno, los sentimientos de impotencia, los estados depresivos, el desacuerdo con la situación de vida actual y la vivencia de duelos, entre otras. ${ }^{24}$

Las personas mayores refieren dificultad para retener informaciones poco significativas, especialmente si deben esforzarse mucho o si en el momento de recibir esa información tienen su foco de atención en alguna otra actividad. También expresan tener problemas en la organización secuencial de la información recién llegada, así como en la capacidad para sintetizar. La memoria a largo plazo, o memoria remota, parece estar bien conservada, los ancianos recuerdan situaciones y hechos antiguos, pero también acontecimientos nuevos almacenados en su memoria remota. Son capaces de evocar con detalle, hechos que tuvieron lugar en otra época, época seguramente significativa en su historia de vida. La memoria remota permite recordar y conservar el vocabulario, las experiencias, los recuerdos y mucha más información útil sobre el mundo que les rodea y sobre sí mismos. Es importante tener presente que la memoria visual se conserva intacta durante más tiempo que la memoria auditiva o que las relaciones temporoespaciales. ${ }^{24}$

La memoria sola no tiene ningún significado si no va acompañada del mantenimiento de la actividad mental. Utilizar medios simples como listas, agendas, notas, calendarios, entre otras, permite recordar a las personas mayores sus ocupaciones, responsabilidades o actividades, sin representar para ellos graves inconvenientes. ${ }^{23,24}$

\section{I mportancia de mantener la actividad física en el adulto mayor}

Si se quiere hacer del envejecimiento una experiencia positiva, una vida más larga debe ir acompañada de oportunidades continuas de salud, participación y seguridad. La Organización Mundial de la Salud (OMS) utiliza el término "Envejecimiento Activo" para expresar el proceso con el que se consigue este objetivo. ${ }^{25,26}$

¿Qué es el "Envejecimiento Activo"? Es el proceso de optimización de las oportunidades de sal ud, participación y seguridad con el fin de mejorar la calidad de vida a medida que las personas envejecen. ${ }^{25,26}$ El término "envejecimiento activo" fue adoptado por la OMS a finales de los años 90 con la intención de transmitir un 
mensaje más completo que el de "envejecimiento saludable" y reconocer los factores que junto a la atención sanitaria afectan la manera de envejecer de los individuos y las poblaciones. ${ }^{27}$

El envejecimiento activo se aplica tanto a los individuos como a los grupos de población, permite a las personas realizar su potencial de bienestar físico, social y mental a lo largo de todo su ciclo vital y participar en la sociedad de acuerdo con sus necesidades, deseos y capacidades, que a su vez les proporciona protección, seguridad y cuidados adecuados cuando necesitan asistencia. ${ }^{25,26}$

El término "activo" hace referencia a una participación contínua en las cuestiones sociales, económicas, culturales, espirituales y cívicas, no solo a la capacidad para estar físicamente activo o participar en actividades manuales. Las personas mayores que se retiran del trabajo y las que están enfermas o viven en situación de discapacidad, pueden seguir contribuyendo activamente con sus familias, sus semejantes, su comunidad y su nación.

El envejecimiento activo trata de ampliar la esperanza de vida saludable y la calidad de vida para todas las personas a medida que envejecen, incluyendo aquellas personas frágiles, con discapacidad o que necesitan asistencia.

El envejecimiento tiene lugar dentro del contexto de los demás: los amigos, los compañeros de trabajo, los vecinos y los miembros de la familia. Por ello, la interdependencia y la solidaridad intergeneracional, dar y recibir de manera recíproca entre individuos, así como entre generaciones de viejos y de jóvenes, son principios importantes del envejecimiento activo. ${ }^{25,26}$ Por lo tanto, el envejecimiento activo debe considerarse como el objetivo primordial de la sociedad y de los responsables políticos, en un intento de mejorar la autonomía, la salud y la productividad de los mayores. ${ }^{25,26} \mathrm{El}$ niño de ayer es el adulto de hoy y será la abuela o el abuelo de mañana. La calidad de vida que disfrutarán como abuelos dependerá de los riesgos y las oportunidades que experimenten a través de toda su vida, así como de la forma en que las generaciones sucesivas proporcionen ayuda y soporte mutuos cuando sean necesarios. ${ }^{25,26}$

La sociedad debe apoyar una "cultura del envejecimiento activo y saludable" que permita que los mayores: ${ }^{25}$

- Sufran menos por las discapacidades relacionadas con las enfermedades crónicas,

- necesiten una atención sanitaria y social menor,

- no padezcan situaciones de soledad y mantengan su independencia y una buena calidad de vida, y que

- sigan participando en el ámbito económico, social, cultural y político, tanto a través de trabajos remunerados como de colaboraciones sin remunerar.

\section{La actividad física y el adulto mayor}

El comportamiento sedentario aumenta con la edad ${ }^{12}$ y es un importante factor de riesgo para trastornos que incluyen enfermedades del corazón, obesidad y diabetes. ${ }^{28}$ En los países desarrollados, el $15 \%$ de la población tiene al menos 65 años de edad ${ }^{1}$ y en Australia, la proporción se espera que alcance el $25 \%$ en $2051 .{ }^{29}$ El resultado de una mayor prevalencia de los trastornos asociados con la inactividad hace de la 
iniciación y el mantenimiento de la actividad física en los adultos mayores una prioridad. ${ }^{12}$

La participación periódica en actividades físicas moderadas puede retrasar el declive funcional y reducir el riesgo de padecer enfermedades crónicas tanto en los ancianos sanos como en aquellos que las sufren. Un estilo de vida activo mejora la salud mental y suele favorecer los contactos sociales. El hecho de mantenerse activo puede ayudar a las personas mayores a conservar la mayor independencia posible durante un mayor período, además de reducir el riesgo de caídas. Por lo tanto, existen ventajas económicas en el hecho de que las personas mayores permanezcan activas físicamente, entre ellas, la reducción considerable en los gastos médicos. ${ }^{25}$ No obstante, una gran proporción de personas mayores lleva una vida sedentaria en la mayoría de los países.

\section{Limitación funcional vs. actividad física}

Los cambios morfológicos que se presentan al envejecer, tienen gran variabilidad con respecto a la edad de comienzo, las estructuras comprometidas, el sexo del individuo y su estilo de vida. La discapacidad se define como el equilibrio negativo entre las capacidades de una persona y los requisitos de su entorno, se suele medir con cuestionarios personales sobre la dificultad que uno encuentra para llevar a cabo varias actividades, como andar, ir a comprar, cocinar y cuidarse. ${ }^{30}$

Como se planteo anteriormente, el envejecimiento se asocia con alteraciones en la composición corporal, incluye una reducción de la masa corporal magra y un aumento de la de masa corporal grasa. La disminución de la masa corporal magra debida al envejecimiento, se produce principalmente como consecuencia de la pérdida de la masa muscular del esqueleto. La pérdida de la masa muscular relacionada con la edad se llama sarcopenia.

La sarcopenia reduce la tasa metabólica basal y la fuerza muscular, que puede conducir a limitaciones funcionales y puede resultar en un menor nivel de actividad física. Esta reducción en la tasa metabólica, así como un reducido nivel de actividad física, conduce a la disminución de la energía en las personas mayores. Si la reducción de requerimiento de energía no se corresponde con el valor energético, lleva al aumento de peso.

La obesidad abdominal en los ancianos se asocia con enfermedades cardiovasculares, la diabetes mellitus tipo 2 y el cáncer. Por otro parte, si se reduce la actividad física y se empareja con una ingesta reducida de alimentos, la incorporación de micronutrientes al organismo puede llegar a ser insuficiente y pondrá en peligro su estado nutricional.

Por lo tanto, el ejercicio suficiente y moderado es crucial en la población de edad avanzada porque, en primer lugar, puede ayudar a prevenir que los adultos mayores se conviertan en frágiles mediante la mejora de la masa muscular, la fuerza muscular y, la densidad ósea, ${ }^{31}$ el fortalecimiento del tejido conectivo y el aumento de la flexibilidad, por consiguiente, mejora la capacidad de hombres y mujeres mayores.; en segundo lugar, puede prevenir el sobrepeso y la obesidad.

Además de su efecto sobre la composición corporal, la actividad física regular, también ha sido reconocida como una estrategia importante para prevenir muchas enfermedades crónicas como la diabetes mellitus tipo 2, enfermedades coronarias y cardiacas y la osteoporosis, entre otras. ${ }^{32}$ Existe también mejora del autoconcepto, la autoestima y la imagen corporal, a la vez que se asocia con la disminución del estrés, 
la ansiedad, el insomnio, el consumo de medicamentos y una mejora de las funciones cognitivas y de socialización. ${ }^{33}$

Al igual que los beneficios fisiológicos de la actividad física en el organismo, las evidencias científicas muestran que existen menos alteraciones en las funciones cognitivas de los individuos que realizan actividad física regular. ${ }^{33}$

Estos hechos sugieren que el proceso cognitivo es más rápido y más eficiente en individuos físicamente activos por mecanismos directos: mejora de la circulación cerebral y en la síntesis y degradación de neurotransmisores, y en los mecanismos indirectos como: disminución de la presión arterial, disminución en el plasma de las concentraciones de lipoproteínas de baja densidad (LDL), disminución de las concentraciones de triglicéridos, e inhibición de la agregación plaquetaria, así como el retraso de la limitación física y funcional en los casos que los adultos mayores lleguen a presentar alteraciones cognitivas.

\section{Actividad física y demencia}

La actividad física puede beneficiar la salud cognitiva mediante los beneficios observados en el sistema cardiovascular, que se extienden al sistema cerebrovascular, o mediante el incremento de la neurogénesis, la mejora de la citoarquitectura cerebral (vasos sanguíneos, dendritas, microglia) y de las propiedades electrofisiológicas, el aumento de los factores de crecimiento cerebrales, y una disminución de la formación de las placas amiloides en la enfermedad de Alzheimer. ${ }^{33}$

Mantener la salud cognitiva en la vejez es una prioridad de salud pública, tanto para personas mayores sanas como para adultos con enfermedades tales como demencias, ya que al realizar actividad física con regularidad disminuye la presentación de los síntomas que generan discapacidad física, haciendo al adulto más funcional por mayor tiempo, lo cual repercute positivamente sobre el cuidador y la sociedad en general.

A pesar de que hechos recientes apuntan a que el ejercicio puede mejorar las funciones cognitivas e incluso proporcionar una disminución del riesgo de padecer determinados tipos de demencia, se precisan más estudios que corroboren estos resultados, pues la evidencia no es totalmente uniforme en los resultados para la reducción del riesgo. ${ }^{34}$

\section{Fisioterapia y prevención de caídas en el adulto mayor}

A medida que se rebasan los 65 años aumenta progresivamente la probabilidad que en los próximos años aparezca una limitación en la funcionalidad. Por ello es tan importante el papel de la geriatría en tratar de prevenir o minimizar esta pérdida de independencia para las actividades de la vida diaria, muy especialmente en relación con los adultos mayores más frágiles. Las caídas en pacientes de edad avanzada a menudo se producen cuando se realizan varias tareas simultáneamente. Los resultados de algunos estudios muestran que la actividad cognitiva concurrente, afecta el equilibrio en las personas mayores. ${ }^{35-40}$

Del mismo modo, el rendimiento cognitivo se reduce por la actividad física simultánea. ${ }^{37,40,41}$ Sin embargo, varios estudios sugieren que algunos ejercicios de doble tarea podrían estar asociados con un mayor equilibrio, porque el control postural se garantiza en el nivel sensorio motor. 
La fisioterapia ayuda a promover, prevenir, curar y recuperar la salud de los individuos, estimulando su independencia. Aplica los métodos y técnicas basadas en un profundo conocimiento del funcionamiento del cuerpo humano, sus movimientos, funciones y su interacción con el entorno. Así, varios programas de fisioterapia destinados a prevenir las complicaciones de la osteoporosis se han diseñado en los últimos 10 años. Estos programas buscan mantener la masa ósea o disminuir el riesgo de caídas. ${ }^{42}$

Los programas diseñados para la prevención de caídas son similares a los programas destinados a combatir los efectos del envejecimiento. Según la literatura, ${ }^{42,43}$ la mayoría de los programas se basan en ejercicios que aumentan la fuerza muscular e incrementan la movilidad de las articulaciones en las extremidades inferiores, la capacidad aeróbica y el equilibrio. De esta forma, los programas de prevención de caídas están diseñados para mejorar el equilibrio mediante el incremento de la fuerza, la resistencia, la flexibilidad, la capacidad aeróbica y la función.

Los fisioterapeutas que trabajan con usuarios geriátricos, lo hacen junto con otros profesionales de la salud para abordar los complejos aspectos de esta población, tanto en las clínicas como en el domicilio del paciente. Tratan los problemas de discapacidad generados por enfermedadades como el parkinson, alzheimer, artritis, fracturas, osteoporosis y problemas cardiorrespiratorios, entre otros y promueven el movimiento y la independencia mediante ejercicios de fuerza y resistencia, técnicas de relajación, manejo del dolor, prescripciones de adaptación de equipamiento y ayudas para la movilidad y otros cuidados propios.

Los resultados de las encuestas realizadas por diferentes investigadores, ${ }^{44,45}$ determinan que los programas de ejercicios para pacientes con osteoporosis persiguen objetivos similares a los diseñados para las personas de tercera edad. De igual forma, se puede confirmar que el ejercicio apropiado es eficaz para mejorar el equilibrio en las personas de edad avanzada. ${ }^{46}$

Las políticas públicas y las intervenciones de la comunidad para reducir el riesgo de caídas o de origen cardiovascular en los países industrializados, han hecho hincapié en la participación en el ejercicio. ${ }^{12}$ Así, existen varios tipos de actividades que pueden y deben ser promovidos en los adultos mayores, entre ellos, las actividades aeróbicas y el entrenamiento de la fuerza muscular, masa ósea y equilibrio.

Actividades aeróbicas. Se recomienda la realización de actividades de bajo impacto como la caminata, el ciclismo o pedaleo en la bicicleta estática, la natación, la hidrogimnasia, el subir escaleras, el baile, el yoga y la gimnasia aeróbica de bajo impacto. Estas actividades son preferibles a las llamadas de alto impacto, como trotar, correr, o practicar deportes con saltos, como el voleibol o baloncesto y gimnasia aeróbica de alta impacto, que tienen alta incidencia de lesiones en la personas de la tercera edad, así pues, es ideal realizar la actividad física en un ambiente agradable, al aire libre si es posible y preferiblemente con varias personas para incentivar la socialización entre los individuos.

Entrenamiento de la fuerza muscular, masa ósea y equilibrio. Un aspecto fundamental del programa de ejercicio es el fortalecimiento de la musculatura buscando aumentar la masa y la fuerza muscular para evitar una de las principales causas de incapacidad y de caídas, sin olvidar mejorar el equilibrio que repercute directamente en el mantenimiento eficaz del patrón de marcha e influye en que no se produzcan caídas. Se sabe también que el entrenamiento de resistencia muscular aumenta el metabolismo en reposo, aumenta el gasto calórico, y la masa libre de grasa, ${ }^{48}$ mejora la tolerancia a la glucosa y mejora la densidad ósea, la masa muscular, la fuerza y el 
equilibrio, por lo que disminuye el riesgo de fracturas por osteoporosis. El ejercicio físico se convierte así en una terapia antienvejecimiento.

\section{El ejercicio como terapia antienvejecimiento}

El ejercicio físico, practicado de manera apropiada, es la mejor herramienta disponible hoy día, para retrasar y prevenir las consecuencias del envejecimiento así como para fomentar la salud y el bienestar de la persona. De hecho, el ejercicio físico ayuda a mantener el adecuado grado de actividad funcional para la mayoría de las funciones orgánicas. De manera directa y específica, el ejercicio físico mantiene y mejora la función muscular esquelética, osteoarticular, cardiocirculatoria, respiratoria, endocrino, metabólica, inmunológica y psiconeurológica.

Realizar ejercicio físico de manera regular reduce el riesgo de desarrollar alteraciones o incluso de morir de lo que hoy día son las principales y más graves causas de morbilidad y mortalidad en los países occidentales. ${ }^{47}$ El ejercicio practicado de manera regular y con la intensidad adecuada (someter al organismo a un programa de entrenamiento), contribuye a mejorar la capacidad funcional de múltiples sistemas orgánicos, que es, precisamente, lo que persiguen los atletas cuando entrenan. No obstante, es preciso resaltar que el momento y la intensidad de las sesiones de entrenamiento tienen que estar perfectamente adaptadas a las características del sujeto, deben ir seguidas del adecuado período de regeneración y, para que se produzca esa mejora de la capacidad funcional, deben aplicarse en el momento adecuado del período de adaptación al esfuerzo o supercompensación. Si el estímulo que representan las sesiones de entrenamiento no se aplica adecuadamente, en el momento oportuno y respetando los períodos de descanso y adaptación, el efecto producido puede ser incluso el contrario al pretendido, esto es, una pérdida de la capacidad funcional. ${ }^{48}$

\section{Beneficios de la actividad física y del ejercicio}

La actividad física es una acción de promoción de la salud. El consenso de los Institutos Nacionales de Salud ${ }^{49}$ define la actividad física como "Movimiento corporal producido por el esqueleto y los músculos, que requiere gasto de energía y produce beneficios progresivos en la salud", en tanto que el ejercicio se describe como "Un movimiento corporal estructurado, planificado y repetitivo, realizado para mejorar o mantener uno o más componentes de aptitud física."

De manera indirecta, la práctica de ejercicio tiene efectos beneficiosos en la mayoría, si no en todas las funciones orgánicas, por lo que contribuye a mantener la funcionalidad e incluso a mejorarla. Dado que la pérdida de funcionalidad que se produce con la edad es, precisamente, la principal consecuencia del envejecimiento, el efecto del ejercicio puede ser considerado como una verdadera terapia que lucha contra las inevitables consecuencias del proceso de envejecimiento. ${ }^{48}$

Existe un gran número de beneficios generados a partir de la práctica de actividad física o ejercicio regular, dentro de los que se encuentran: ${ }^{28,34,51,52}$

- Prevención y reducción de los riesgos de enfermedades como: obesidad, diabetes mellitus, osteoporosis, cáncer de colon, enfermedad coronaria, endometriosis posmenopáusica, depresión y accidentes relacionados con caídas.

- Incremento de la longevidad y disminución de la discapacidad. 
- Reducción de la ansiedad y el estrés.

- Aumento de la confianza y de la autoestima.

- Mantenimiento de un peso saludable y mejoría en la capacidad física.

- Fortalecimiento de los músculos y los huesos.

- Mejora del estado de ánimo.

- Mejora en patrones de sueño.

- Minimiza los cambios biológicos relacionados con el envejecimiento.

- Revierte los síndromes por desuso.

- Previene las enfermedades crónicas.

- Controla y mejora los síntomas de enfermedades crónicas.

- Maximiza la salud psicológica.

De manera más específica a nivel del sistema músculo esquelético, ${ }^{28,53}$ los beneficios del ejercicio o la actividad física se aprecian en:

- Incremento de la densidad mineral en los adolescentes, su mantenimiento en los adultos jóvenes y retardo de su declinación en los adultos mayores.

- Retardo de la progresión de la osteoporosis, aunque no revierte la pérdida avanzada de hueso.

- Retraso de la osteoartritis.

- Beneficio específico del ejercicio de alto impacto en el hueso.

- Reducción del riesgo de discapacidad funcional en los adultos mayores.

- Mejora de la fuerza y la flexibilidad.

- Aumento del equilibrio, la movilidad, y el desempeño físico funcional.

A nivel de la capacidad funcional, el ejercicio regular o la actividad física logran: ${ }^{28}$

- Reducir el riesgo de caídas debido a un aumento de la fuerza, la flexibilidad y el equilibrio.

- Reducir el riesgo de fracturas.

- Facilitar la rehabilitación de enfermedades agudas y crónicas.

- Disminuir el tiempo de reacción, manteniendo la perfusión cerebral y la cognición. 
En individuos con enfermedades cardiovasculares, el ejercicio consigue: ${ }^{28}$

- Prevenir los accidentes cerebrovasculares.

- Contribuir al tratamiento de la enfermedad vascular periférica.

- Disminuir el sedentarismo y la baja aptitud física, los cuales constituyen los principales factores de riesgo para la enfermedad coronaria en hombres y mujeres.

- Disminuir el riesgo de morir por enfermedad coronaria comparadas con las personas fumadoras.

- Modificar otros factores de riesgo cardiovasculares como disminución de la tensión arterial y los cambios en los perfiles de los lípidos.

El ejercicio o la actividad física regular en personas con sobrepeso y obesidad logra: ${ }^{28}$

- Maximizar la pérdida de grasa combinada con una dieta adecuada.

- Aumentar la masa muscular magra.

- Mejorar la aptitud física.

En personas con diabetes: ${ }^{28}$

- La actividad física regular ayuda al mejor control de los niveles de glucosa.

- Minimiza el factor de riesgo para desarrollar diabetes tipo 2.

La práctica de ejercicio o actividad física regular genera un bienestar psicológico al reducir la ansiedad y mejorar la autopercepción, igualmente ayuda a aliviar el estrés y mejora el sueño. ${ }^{28}$

\section{CONSI DERACI ONES FI NALES}

El mejoramiento en el control de las enfermedades crónicas ha generado un incremento en el número de personas mayores, que pueden sufrir alteraciones en su condición de salud, propias del proceso de envejecimiento.

La clave para contar con una población sana es el denominado por la OMS "Envejecimiento Activo", el cual incluye estrategias de promoción de salud y prevención de las deficiencias y las discapacidades e involucra programas de ejercicio físico o actividad física practicados de manera regular.

El ejercicio físico o la actividad física practicados de manera regular y planificado específicamente para personas mayores, está asociado con un menor riesgo de morbilidad y mortalidad y con una mejora en la calidad de vida de esta población, secundario al efecto protector cardiovascular que genera, el cual disminuye el riesgo de sufrir un infarto de miocardio y de desarrollar diabetes tipo 2. 
Por otra parte, el ejercicio o la actividad física regular han mostrado ser eficaces en el incremento de la densidad mineral ósea, el mejoramiento del equilibrio con la subsecuente reducción de caídas que en esta etapa de la vida pueden terminar en pérdida de la funcionalidad física, de la independencia personal, ser causa de invalidez e incluso llevar a la muerte. Además, la literatura informa que el ejercicio o la actividad física practicada de manera regular, mejora la función cognitiva, reduciendo el riesgo de padecer demencia y enfermedad de Alzheimer.

De manera general, el ejercicio físico o la actividad física, mantienen y mejoran la función músculo esquelética, osteoarticular, cardiocirculatoria, respiratoria, endocrino metabólica, inmunológica y psiconeurológica de las personas mayores. Igualmente tiene efectos beneficiosos en la mayoría de las funciones orgánicas del adulto mayor, contribuyendo a mejorar su funcionalidad, lo cual es sinónimo de mejor salud, mejor respuesta adaptativa y mayor resistencia ante la enfermedad. ${ }^{54}$

Es importante considerar que los programas de ejercicio o actividad física dirigidos a personas mayores deben orientarse al mejoramiento de sus condiciones físicas pero principalmente al mejoramiento del equilibrio, el mantenimiento o favorecimiento de la independencia funcional y el mejoramiento de la calidad de vida de esta población y de sus cuidadores.

Finalmente, se debe reflexionar acerca de invertir en programas de ejercicio o actividad física de calidad dirigido a la población mayor a través de profesionales calificados con amplio dominio de estos temas, lo que puede generar un ahorro considerable para la sanidad del país, al disminuir costos por caídas, accidentes cerebrovasculares, infartos del miocardio o por el padecimiento de problemas de origen músculo esquelético y de enfermedades crónicas propias de esta etapa de la vida.

\section{REFERENCI AS BI BLI OGRÁFICAS}

1. McMurdo MET. A healthy old age: realistic or futile goal? $\mathrm{Br}$ Med J. 2000; 321: 114951.

2. Carville SF, Perry MC, Rutherford OM, Smith IC, Newham DJ. Steadiness of quadriceps contractions in young and older adults with and without a history of falling. Eur J App Physiol. 2007; 100(5):527-33.

3. Macaluso A, De Vito G. Muscle strength, power and adaptations to resistance training in older people. Eur J App Physiol. 2004;91(4):450-72.

4. Hunter GR, McCarthy JP, Bamman MM. Effects of resistance training on older adults. Sports Med. 2004;34(5):329-348.

5. Shaffer SW, Harrison AL. Aging of the somatosensory system: a translational perspective. Physical Ther. 2007;87(2):193-207.

6. Doherty TJ. Invited review: aging and sarcopenia. J App Physiol. 2003;95(4):171727.

7. Orr R, Raymond J, Fiatarone M, Singh M. Efficacy of progressive resistance training on balance performance in older adults: a systematic review of randomized controlled trials. Sports Med. 2008;38(4):317-43. 
8. Yusuf HR, Croft JB, Giles WH. Leisure-time physical activity among older adults: United States, 1990. Arch Intern Med. 1996; 156: 1321-6.

9. Heath GW, Smith JD. Physical activity patterns among adults in Georgia: results from the 1990 Behavioral Risk Factor Surveillance System. South Med J. 1994; 87:435-9.

10. Davis MA, Neuhaus J M, Moritz DJ, Lein D, Barclay JD, Murphy SP. Health behaviors and survival among middle-aged and older men and women in the NHANES I Epidemiologic Follow-up Study. Prev Med. 1994;23:369-76.

11. Department of Health and Human Services. Physical activity and health: a report of the Surgeon General. Atlanta, GA: U.S. Department of Health and Human Services; 1996.

12. Owen N, Bauman A. The descriptive epidemiology of a sedentary lifestyle in adult Australians. Internat J Epidemiol. 1992;21:305-10.

13. Greef de MHG, Stevens M, Bult $P$, Lemmink KAPM. Groningen Active Living Model: Manual. Haarlem: De Vrieseborch; 1997.

14. Sports Council and Health Education Authority. Allied Dubar National Fitness Survey: a report on activity patterns and fitness levels. London: Sports Council and Health Education Authority; 1992.

15. Christmas C, Andersen RA. Exercise and older patients: guidelines for the clinician. J Amer Geriatrics Soc. 2000;48:318-24.

16. Ivy JL. Role of exercise training in the prevention and treatment of insulin resistance and noninsulin-dependent diabetes mellitus. Sports Med. 1997;24:321-36.

17. Ernst E. Exercise for female osteoporosis. A systematic review of randomized clinical trials. Sports Med. 1998; 25:359-68.

18. Carlson JE, Ostir GV, Black SA, Markides KS, Rudkin L, Goodwin JS. Disability in older adults. Physical activity as prevention. Behav Med. 1999;24:157-68.

19. Goméz Montes JF. Valoración Integral de la Salud del Anciano, ED Manizales: Artes Gráficas Tizan; 2002.

20. Hombres, envejecimiento y salud. Unidad del envejecimiento y el curso de la vida. Ginebra: Organización Mundial de la Salud; 2001.

21. Velásquez M, Prieto B, Contreras R. El Envejecimiento y los Radicales Libres. Ciencias. 2004; 75: 36-43.

22. American Psychiatric Association. Diagnostic and statistical manual of mental disorders (DSM-IV-TR). 4 ed. Text revision. Washington, D. C.: APA; 2000.

23. Rockwood K, Middleton L. Physical activity and the maintenance of cognitive Function. Alzheimer's Dementia. 2007; 3:S38S44.

24. Noriega Borge MJ, García Hernández M, Torres Egea MP. Proceso de envejecer: cambios físicos, cambios psíquicos, cambios sociales [tesis]. Comunidad Autónoma de Cantabria: Universidad de Cantabria; 2005. 
25. Organización Mundial de la Salud. Envejecimiento activo: un marco político. Rev Esp Geriatr Gerontol. 2002; 37(S2):74-105.

26. Organización Mundial de la Salud. Growing Older. Staying Well. Ageing and Physical Activity in Everyday Life. Ginebra: OMS; 1998.

27. Kalache A, Kickbusch I. A global strategy for healthy ageing. World Health. 1997;4-5.

28. Department of Health and Human Services, U.S. Physical Activity and Health: A Report of the Surgeon General. Atlanta GA, U.S.: Department; 1996.

29. Australian Bureau of Statistics Cat 3222.0. Populations Projections 2006 to 2101 [Internet]. Australian Government; 2005 [cited 2012 J un 6]. Available from: http://www.abs.gov.au/Ausstats/abs@.nsf/mf/3222.0

30. Verbrugge LM, J ette AM. The disablement process. Soc Sci Med. 1994;38:1-14.

31. Dock J, Henderson N, Price R. Exercise and bone mineral density in mature female athletes. Med Sci Spots Exerc. 1997;29 (3):291-6.

32. Schuita AJ. Physical activity, body composition and healthy ageing exercice, composition corporelle et vieillissement. Sci Sports. 2006;21:209-13.

33. Rolland $Y$, Van Kan GA, Vellas B. Physical activity and Alzheimer's disease: from prevention to therapeutic perspectives. J Am Med Dir Assoc. 2008; 9: 390-405.

34. Varo Cenarruzabeitia JJ. Beneficios de la actividad física y riesgos del sedentarismo. Med Clín (Barc). 2003; 121(17):665-72.

35. Andersson G, Yardley L, Luxon L. A dual task study of interference between mental activity and control of balance. Am J Otol. 1998; 19:632-7.

36. Brown LA, Shumway-Cook A, Woollacott MH. Attentional demands and postural recovery: the effects of aging. J Gerontol A Biol Sci Med Sci. 1999; 54: M165-M171.

37. Hauer K, Marburger C, Oster P. Motor performance deteriorates with simultaneously performed cognitive tasks in geriatrics patients. Arch Phys Med Rehabil. 2002;83:217-23.

38. Maylor EA, Wing AM. Age differences in postural stability are increased by additional cognitive demands. J Gerontol Psychol Sci. 1996; (S1B): 143-54.

39. Shumway-Cook A, Woollacott M, Kerns KA, Balwin H. The effects of 2 types of cognitive tasks on postural stability in older adults with or without history of falls. J Gerontol A Biol Med Sci. 1997; (S2A: M):232-40.

40. Teasdale N, Simoneau M. Attentional demands for postural control: the effects of aging and sensory reintegration. Gait Posture. 2001;14:203-10.

41. Marsh AP, Geel SE. The effect of age on the attentional demands of postural control. Gait Posture. 2000; 12: 105-13.

42. Turner PA. Osteoporosis- its causes and prevention: an update. Physiother Theory Pract. 2000; 16: 135- 49.

http://scielo.sld.cu 
43. Brown AP. Reducing falls in elderly people: a review of exercise interventions. Physiother Theory Pract. 1999; 15:59-68.

44. McGilvray L, Cott CA. A key informant survey of osteoporosis exercise programs in Ontario. Physiother Can. 2000; spring: 146-52.

45. Sran MM, Khan KM. Physiotherapy and osteoporosis: practice behaviours and clinician's perceptions- a survey. Man Ther. 2005; 10:21-7.

46. Fordham R, Hodkinson C. A cost benefit analysis of open access to physiotherapy for GPs: discussion paper 29. ED. University of York Centre for Health Economics. York: Centre for Health; 1988.

47. Department of Health and Human Services. U.S. Physical activity fundamental to Preventing disease. Office of the assistant secretary for planning and evaluation [Internet]. Atlanta GA, U.S.: Department; 2002 Jun [cited 2012 Jun 6]. Available from: http://aspe.hhs.gov/health/reports/physicalactivity/physicalactivity.pdf

48. Castillo MJ, Ortega FB, Ruiz J. Mejora de la forma física como terapia antienvejecimiento. Med Clín (Barc). 2005; 124(4): 146-55

49. Physical activity and cardiovascular health. NIH Consensus Development Panel on Physical Activity and Cardiovascular Health. JAMA. 1996;276(3):241-6.

50. Dunn AL, Andersen RE, Jackicic JM. Lifestyle physical activity interventions: history, short-and long-term effects, and recommendations (OMS. Estrategia mundial sobre régimen alimentario, actividad física y salud. Mayo, 2004). Am J Prev Med. 1998; 15: 398-412.1

51. World Health Organization. World health report. Geneva: WHO; 2002.

52. Warburton D, Whitney C, Bredin S. Health benefits of physical activity: the evidence. CMAJ . 2006; 174(6):801-9.

53. Lim K-C, Kayser-J ones JS, Waters C, Yoo G. Aging, Health, and Physical Activity in Korean Americans. Geriatric Nursing. 2007;28(2): 112-11.

54. Castillo-Garzón MJ, Ruiz J R, Ortega FB, Gutiérrez A. Anti-aging therapy through fitness enhancement. Clin Interven aging. 2006; 1(3):213-20.

Recibido: 14 de febrero de 2012.

Aprobado: 22 de mayo de 2012.

Nancy Stella Landinez Parra. Departamento Movimiento Corporal Humano. Facultad Medicina Of. 519. Universidad Nacional de Colombia. Ciudad Universitaria. Bogotá, Colombia.

Correo electrónico: nslandinezp@unal.edu.co

http://scielo.sld.cu 